\title{
Féeries
}

Études sur le conte merveilleux, XVII $-\mathrm{XIX}{ }^{\mathrm{e}}$ siècle

\section{Reframing the Early French Fairy Tale. Marvels \& Tales, Journal of Fairy Tales Studies}

Vol. 19, number 1, 2005, Wayne State University Press, Detroit, Michigan, $156 \mathrm{p}$.

Jean-François Perrin

\section{OpenEdition}

Journals

Édition électronique

URL : http://journals.openedition.org/feeries/503

DOI : $10.4000 /$ feeries.503

ISSN : 1957-7753

Éditeur

UGA Éditions/Université Grenoble Alpes

Édition imprimée

Date de publication : 1 octobre 2007

Pagination : 263-267

ISBN : 978-2-84310-101-4

ISSN : 1766-2842

Référence électronique

Jean-François Perrin, «Reframing the Early French Fairy Tale. Marvels \& Tales, Journal of Fairy Tales

Studies », Féeries [En ligne], 4 | 2007, mis en ligne le 18 décembre 2013, consulté le 24 septembre

2020. URL : http://journals.openedition.org/feeries/503 ; DOI : https://doi.org/10.4000/feeries.503

Ce document a été généré automatiquement le 24 septembre 2020.

(c) Féeries 


\section{Reframing the Early French Fairy Tale. Marvels \& Tales, Journal of Fairy Tales Studies}

Vol. 19, number 1, 2005, Wayne State University Press, Detroit, Michigan, $156 \mathrm{p}$.

Jean-François Perrin

1 Cette livraison de la revue Marvels \& Tales est consacrée à certaines problématiques émergentes aux États-Unis concernant le contes de fées français de la première vague. Coordonné par Holly Tucker, professeur à Vanderbilt University, et auteur notamment de Pregnant Fictions: Childbirth and the Fairy Tale in Early Modern France (2003) ${ }^{1}$, ce numéro interroge la spécificité "française » du genre, reprend le problème de ses « sources " écrites, et propose des pistes pour aborder sous un nouveau jour certains thèmes rebattus comme la violence ou le monstrueux, en décloisonnant culturellement son approche critique. On y trouve également un essai de bilan de la bibliographie récente sur la période considérée.

2 L'étude la plus originale de cet ensemble est sans doute celle que signe Ruth B. Bottigheimer. Poursuivant une enquête commencée dans son précédent livre : Fairy Godfather: Straparola, Venice, and the Fairy Tale Tradition ${ }^{2}$, elle remet radicalement en cause - après d'autres mais avec de nouveaux arguments -, la thèse des sources au moins partiellement orales des premiers contes de fées "littéraires", thèse qu'elle considère comme indémontrée et indémontrable dans l'état actuel de la documentation. À l'inverse, elle propose d'orienter la recherche vers les seules sources littéraires: reconstituant l'histoire éditoriale de la diffusion française des Nuits facétieuses de Straparole, elle y voit la base matérielle réelle de l'introduction du conte de fées en France, du moins selon la définition qu'elle lui donne: soit scénario de «restauration" (la magie permet au prince ou à la princesse de retrouver le statut royal qu'ils ont perdu), soit scénario d'« ascension » (un(e) enfant pauvre accède au statut princier, grâce à des adjuvants féeriques). Son propos est en effet centré sur ce type de contes à personnel aristocratique dominant, excluant le personnel plus 
populaire et surtout plus mêlé du conte de tradition orale. Selon elle, c'est le commerce des livres qui introduit le conte de fées en France à partir de la fin du $\mathrm{XVI}^{\mathrm{e}}$ siècle; si transmission orale il y eut éventuellement de cette matière (au sein du cercle familial), elle est plus vraisemblablement issue de lectures que d'une improbable tradition populaire du conte de fées; au passage, se trouve écorné à la lumière de la documentation historique moderne, le mythe de la nourrice-conteuse dont les occupations et les conditions de vie rurales excluent selon l'auteur qu'elle ait véritablement existé. Sur le second point, l'article présente une étude précise et documentée de la diffusion des Nuits facétieuses dans la traduction de P. de Larivey dont la première édition est publiée à Lyon dès 1560 selon une étude peu connue de Louis Morin : «Les trois Pierre de Larivey ", Mémoires de la société académique du département de l'Aube $\mathrm{n}^{\circ} 97$ (1935-1936), non prise en compte dans la récente réédition de cette traduction chez Corti. Il apparait que l'ouvrage est très rapidement devenu une sorte de "best seller» entre 1560 et 1615, particulièrement à Paris, tandis qu'à Lyon les réimpressions se succèdent entre 1577 et 1611, jusqu'à ce que sa mise à l'Index en 1615 bloque ce processus. Arguant de ce que ce recueil est le seul du corpus de nouvelles imitées du Décaméron publiées en France à cette époque, à inclure des récits repérable après coup comme matrices du conte de fées de la fin du XvII ${ }^{e}$ siècle, et s'appuyant sur le témoignage bien connu de $\mathrm{M}^{\mathrm{me}}$ de Murat à ce sujet dans l'« Avertissement » de ses Histoires sublimes et allégoriques, Ruth B. Bottigheimer affirme que les principaux conteurs de la première vague ont largement travaillé sur Straparole, et propose de lancer un programme de recherche sur les spécificités stylistiques de ces adaptations. Elle avance également que la prise en compte de son hypothèse modifie l'approche générale concernant la diffusion du conte de fées en Europe, qu'il faut étudier, selon elle, à partir d'une approche historienne des réseaux de commerce de librairie plutôt qu'en termes de diffusion orale.

3 Tout en saluant le renouvellement indéniable que cette problématique apporte au vieux problème des sources des premiers conteurs littéraires, on peut tout de même se demander ce qui permet d'une part de révoquer absolument les quelques témoignages contemporains sur des faits de transmission orale, et surtout de privilégier à ce point (du moins dans cet article) l'influence de Straparole; nul dans la critique moderne ne songe à nier son impact littéraire sur les premiers contes de fées (la démonstration en serait fastidieuse: voir entre autres l'édition critique de J.-P. Collinet et l'étude de Michèle Simonsen), mais en composition avec diverses autres sources, entre autres le fameux Pentamerone - sur l'influence duquel Marc Soriano avançait déjà, concernant Perrault, des hypothèses prudentes -, une influence que la critique moderne a démontrée notamment chez $\mathrm{M}^{\text {lle }}$ Lhéritier (R. Robert, 2002, p. 32-133) et fortement suggérée chez $\mathrm{M}^{\mathrm{me}} \mathrm{d}$ 'Aulnoy (N. Jasmin, 2002, p. 83-84), du moins par l'analyse interne, puisque les preuves «externes » font encore défaut concernant les voies concrètes de sa transmission et de sa traduction parmi les écrivains français ${ }^{3}$. Sans négliger enfin ce que les travaux récents sur la naissance de l'opéra français ${ }^{4}$ montrent du contexte favorable créé par le genre de merveilleux cultivé à l'opéra depuis Alceste (1673), pour l'invention littéraire du conte de fées - influence dont témoigne Perrault dans le Parallèle et ailleurs, mais qui surtout se lit (et s'écoute) comme vers à la Quinault et scénographies d'opéra ou de ballet transposées, en bien des contes de $\mathrm{M}^{\mathrm{me}} \mathrm{d}$ 'Aulnoy (dès L'île de la Félicité), de $\mathrm{M}^{\mathrm{me}}$ de Murat et d'autres conteuses encore. Il semble que se soit développé là dès l'origine - et de part et d'autre - un compagnonnage générique dont le présent numéro de Féeries montre d'ailleurs la remarquable fécondité aux XvII 
XVIII ${ }^{\mathrm{e}}$ siècles. Quoi qu'il en soit, les questions et la méthode de R. Bottigheimer permettent d'évaluer avec plus de précision la part du conte merveilleux issu des recueils italiens dans l'invention du conte de fées à la française.

Deux autres articles du numéro reprennent de façon originale et intéressante les questions de la violence et du monstrueux dans le conte de fées. L'intitulé provocateur d'Elizabeth Wanning Harries: "The Violence of the Lambs", ne doit pas laisser méconnaitre la force de sa thèse: elle montre en effet que le terrain central de la violence travaillée par le conte est celui du langage. Son corpus principal pour cet article est constitué de trois contes de $\mathrm{M}^{\mathrm{me}} \mathrm{d}$ 'Aulnoy : Chatte blanche, Serpentin vert et Le Mouton. Mais il inclut aussi la réécriture de Straparole dans Le Prince Marcassin et inclut un regard vers les contes de Grimm en aval, et vers certains contes-types du catalogue Aarne-Thompson (par ex. "The Mouse as a Bride»). La constitution de la démétamorphose en rituel sacrificiel est analysée en termes de renaissance symbolique, mais surtout l'auteur pointe le lien récurrent ici entre la violence sacrificielle et le récit "autobiographique» ensuite délivré par la victime démétamorphosée. Tout se passe comme si la langue du conte chez d'Aulnoy et ses consoeurs, transformait une situation historique de violence symbolique en prise de parole prétendant à l'autonomie critique dans le champ des représentations.

5 L'article de Kathryn A. Hoffmann illustre parfaitement, pour sa part, l'ambition novatrice de ce numéro, en se saisissant de la thématique du monstre et du monstrueux bien reconnue comme élément constitutif du genre, pour proposer une contextualisation originale par rapport à d'autres secteurs de la culture ancienne comme la littérature médicale, les cabinets de curiosité ou les spectacles de foire (une utile bibliographie anglo-saxonne en dresse la cartographie) ${ }^{5}$. Le conte de Babiole (d'Aulnoy), avec sa princesse guenon, est ainsi confronté aux récits et portraits contemporains concernant des cas attestés d'hypertrichosis (développement anormal du système pileux) publiquement produits ou représentés, jouant du clavecin, peignant, maniant plusieurs langues, etc., à propos desquels peut être saisie une interrogation récurrente dans divers discours ou pratiques publiques, sur la fragilité ou la relativité des frontières et partages culturellement reçus, aux confins de la science et du merveilleux, de la norme et du pathologique, de l'observation et du voyeurisme. Du coup, l'extravagance baroque du monde de $\mathrm{M}^{\text {me }} \mathrm{d}$ 'Aulnoy y acquiert la dimension d'une sorte de résonateur ou d'amplificateur remarquable des curiosités et des inquiétudes relatives à l'altérité dans la culture classique. C'est donc à décloisonner le plus largement l'approche du conte de fées, qu'appelle cet article; on peut d'ailleurs signaler de ce côté-ci de l'Atlantique, les ouvertures en direction d'une telle approche proposées, par exemple, dans les travaux d'Aurélia Gaillard ou de Patrick Tort ${ }^{6}$.

On trouvera encore dans cette livraison de Marvels \& Tales, une étude de $\mathrm{M}^{\mathrm{me}}$ Allison Stedman proposant de lire dans la fameuse Histoire d'Hypolite comte de Duglas de $\mathrm{M}^{\mathrm{me}}$ d'Aulnoy, un "manifeste du conte de fées ». L'utilité de cette étude est de mettre en relief certaines corrélations et parallèles structurels ou thématiques entre le cadre romanesque et le fameux conte inséré qu'il contient. En revanche, on s'étonne ici de la non prise en compte du travail fondateur de Jean Mainil : Madame d'Aulnoy et le rire des fée, dont le chapitre II est entièrement consacré à ce roman envisagé dans la perspective d'une écriture ironique jouant, entre autres, sur le différentiel du cadre romanesque et de la forme-conte. $\mathrm{M}^{\mathrm{me}}$ Stedman proposant une lecture presque radicalement inverse, une discussion serrée s'imposait. 
7 Il faut enfin signaler l'important travail du professeur Bérénice Virginie Le Marchand présentant sur près de quarante pages un état récent (mais donné comme « sélectif») de la bibliographie (textes et critiques) concernant le domaine considéré. Les « sources primaires " sont réparties en une quarantaine d'entrées allant de $\mathrm{M}^{\text {me }} \mathrm{d}^{\prime}$ Aulnoy à Voisenon (incluant donc des conteurs du XVIII ${ }^{e}$ siècle). L'actualité des publications évoluant rapidement et la perfection n'étant pas de ce monde, cette utile recension est parfois lacunaire, y compris concernant des éditions de référence; il y manque par exemple les «fables et opuscules pédagogiques» de Fénelon dans le vol. 1 des Euvres en Pléiade (Gallimard, 1983), la bonne édition de la Reine Fantasque de Rousseau dans la même collection (OC de J-J. Rousseau, vol. II, 1964), l'excellente édition critique des Euvres complètes de Crébillon fils chez Garnier-Bordas (1999-2002) ou encore celle des Quatre Facardins d'Hamilton par G. May (Desjonquères, 2001). Dans le domaine de la littérature " secondaire ", on regrettera également quelques oublis ${ }^{7}$ tout en soulignant volontiers la très grande utilité de cette section pour qui souhaite compléter son information sur les courants modernes de la recherche en langue anglaise concernant le conte merveilleux du xvII ${ }^{\mathrm{e}}$ siècle français.

\section{NOTES}

1. Detroit, Wayne State UP, 2003.

2. University of Pennsylvania Press, Philadelphia, 2002.

3. On a une preuve de la circulation du Pentamerone en France dans les premières décennies du XVIIIe siècle, puisque Lenglet-Dufresnoy inclut une édition romaine de 1679 de « Lo Cunto de li Cunti » dans sa Bibliothèque des romans (1734), article XII : « Contes et nouvelles...», p. 309.

4. Voir l'introduction de Christelle Bahier-Porte au présent numéro.

5. Notamment L. Daston et K. Park: Wonders and the Order of Nature (1150-1750), New York, Zone books, 1998. M-H. Huet: Monstrous Imagination, Cambridge, Harvard UP, 1993. K. Pomian : Collectors and Curiosities, Paris \& Venise, 1500-1800. Cambridge, Polity P. 1990.

6. Respectivement Fables, Mythes, contes : l'esthétique de la fable et du fabuleux (1660-1724), Champion, 1996. L'Ordre et les monstres (rééd. Syllepse 1998).

7. Par ex. J-P. Sermain: Métafictions (1670-1730), Paris, Champion, 2002 (compte rendu dans Féeries $\mathrm{n}^{\circ} 1$ ). Voir maintenant du même auteur, Le Conte de fées du classicisme aux Lumières, Paris, Desjonquères, 2005 (compte rendu dans Féeries $n^{\circ}$ 3). Concernant le XVIII ${ }^{\mathrm{e}}$ siècle, je prends la liberté de rappeler les actes du colloque de Grenoble: Le Conte merveilleux au XVIII ${ }^{e}$ siècle: une poétique expérimentale (dir. R. Jomand-Beaudry et J-F. Perrin), Paris, Kimé 2002, et de signaler la publication récente des actes du colloque : Le Conte en ses paroles, la figuration de l'oralité dans le conte merveilleux du Classicisme aux Lumières (dir. A. Defrance et J-F. Perrin), Paris, Desjonquères, 2007. 